\title{
Recent Molecular Assessment of Plasmodium vivax and Plasmodium falciparum Asymptomatic Infections in Botswana
}

Thato Motshoge, ${ }^{1}$ Daniel H. Haiyambo, ${ }^{2}$ Ruth Ayanful-Torgby, ${ }^{3,4}$ Larysa Aleksenko, ${ }^{5}$ Davies Ntebela, ${ }^{6}$ Benoit Malleret, ${ }^{7,8}$ Laurent Rénia, ${ }^{9}$ Elias Peloewetse, ${ }^{1}$ Giacomo Maria Paganotti, ${ }^{10,11,12}$ and Isaac K. Quaye ${ }^{3 *}$

${ }^{1}$ University of Botswana, Department of Biological Science, Gaborone, Botswana; ${ }^{2}$ University of Namibia School of Medicine, Windhoek, Namibia; ${ }^{3}$ Regent University College of Science and Technology, Department of Engineering, Computing and Allied Health Sciences, Accra, Ghana; ${ }^{4}$ Biomedical and Public Health Research Unit, Council for Scientific and Industrial Research-Water Research Institute, Council Close, Accra, Ghana; ${ }^{5}$ University of Lund, Department Clinical Sciences, Lund, Sweden; ${ }^{6}$ National Malaria Program Ministry of Health and Wellness, Gaborone, Botswana; ${ }^{7}$ Department of Microbiology and Immunology, Immunology Translational Research Program, Yong Loo Lin School of Medicine, Immunology Program, Life Sciences Institute, National University of Singapore, Singapore; ${ }^{8}$ Singapore Immunology Network (SlgN), Agency for Science, Technology and Research (A*STAR), Biopolis, Singapore; ${ }^{9} A^{*}$ STAR Infectious Diseases Laboratories, Agency for Science, Technology and Research (A*STAR), Biopolis, Singapore; ${ }^{*}{ }^{*}$ Botswana-University of Pennsylvania Partnership, University of Botswana, Gaborone, Botswana;

${ }^{11}$ Division of Infectious Diseases, Perelman School of Medicine, University of Pennsylvania, Philadelphia, Pennsylvania; ${ }^{12}$ Department of Biomedical Sciences, Faculty of Medicine, University of Botswana, Gaborone, Botswana

\begin{abstract}
In 2016, we reported the presence of Plasmodium vivax in Botswana through active case detection. A realtime PCR was used during a similar study in 10 districts to assess changes in the $P$. vivax prevalence. We assessed 1,614 children (2-13 years of age) for hemoglobin $(\mathrm{Hb} ; \mathrm{g} / \mathrm{dL})$ and Plasmodium parasites. The median age of all participants was 5.0 years $\left(25^{\text {th }}\right.$ percentile, 3 years; $75^{\text {th }}$ percentile, 8 years). The median $\mathrm{Hb}(\mathrm{g} / \mathrm{dL}$ ) level was 12.1 , but $18.3 \%$ of the participants had anemia $(\mathrm{Hb}<11.0 \mathrm{~g} / \mathrm{dL})$; these participants were clustered in the younger than 5 years age group in all districts $(P<0.001)$. The risk of anemia decreased with age 5 years or older (odds ratio [OR], $0.26 ; 95 \%$ confidence interval [Cl], 0.197-0.34; $P<0.001)$. The prevalence rates of Plasmodium parasites were as follows: $P$. vivax, $12.7 \% ; P$. falciparum, $12.7 \%$; P. malariae, $0.74 \%$; and $P$. ovale ( $P$. ovale curtisi), $0.68 \%$. Mixed infection rates were as follows: $P$. falciparum and $P$. vivax, $2.35 \% ; P$. falciparum and $P$. ovale curtisi, $0.56 \% ; P$. vivax and $P$. malariae, $0.06 \%$; and $P$. falciparum and P. malariae, $0.68 \%$. The infections were largely asymptomatic (99.6\%). Using logistic regression, the risk of infection with $P$. vivax was highest in Kweneng East (OR, 6.2; 95\% Cl, 2.9-13.1), followed by South East (OR, 5.6; 95\% Cl, 2.5-12.3) and Ngami (OR, 5.1; 95\% Cl, 2.2-12.0). Compared to the risk of infection for children younger than 5 years, the risk of infection decreased for children 5 years or older in regions with high rates of $P$. vivax and $P$. falciparum infections. $P$. vivax and $P$. falciparum have expanded within the asymptomatic population in Botswana; therefore, careful attention is required for their elimination.
\end{abstract}

\section{INTRODUCTION}

Botswana is a member of the Southern African Development Community (SADC) Elimination 8 (E8) countries that aimed to eliminate malaria by $2018 .{ }^{1}$ Unfortunately, the elimination target could not be achieved because of persistent factors, including the sole focus of the National Malaria Program (NMP) on Plasmodium falciparum ( $P$. falciparum) for elimination, limited human resource capacity, and structural/ logistic impediments that affect disease surveillance.

In 2016, the first report of the presence of asymptomatic $P$. vivax infections in the country was made in a collaborative venture with the NMP. ${ }^{2}$ Between 2015 and 2017, a similar collaboration to perform active case detection of all Plasmodium species that infect humans ( $P$. falciparum, $P$. vivax, $P$. ovale) and $P$. malariae in schools and hospitals located in 10 districts across the country was performed to assist the NMP with the stratified data required for an effective malaria elimination plan. ${ }^{3-5}$ In 2019, the World Health Organization (WHO) noted that the elimination agenda in Botswana was not working and needed to be reviewed with a new approach. ${ }^{6}$ This was a necessary approach to align with the overall global target of malaria eradication. ${ }^{7}$

\footnotetext{
${ }^{*}$ Address correspondence to Isaac K. Quaye, Department of Engineering, Computing and Applied Health Sciences, Regent University College of Science and Technology, Box DS 1636,
} Dansoman, Accra, Ghana. E-mail: isaac.quaye@regent.edu.gh
It has been well-established that as the rates of malaria attributable to $P$. falciparum decrease, the rates of nonfalciparum ( $P$. vivax, $P$. ovale, and $P$. malariae) malaria increase. $^{8,9}$ In addition, $P$. vivax is now well-recognized in Africa, including in Duffy-negative individuals. ${ }^{10}$ Both $P$. malariae and $P$. ovale are commonly found in Africa. However, unlike the two sympatric species of $P$. ovale ( $P$. ovale wallikeri and $P$. ovale curtisi) that have hypnozoites stages similarly to $P$. vivax, it is unknown if $P$. malariae have hypnozoites even though they have long latency periods and can sustain infections through relapses or recrudescences. ${ }^{11,12}$ $P$. malariae is known to have an enhanced capability to sustained infection in humans. ${ }^{13}$ Several reports have shown that asymptomatic infections with all Plasmodium species comprise a significant portion of the burden of disease. ${ }^{11,14,15}$ Therefore, it is reasonable to surmise that a more effective approach to malaria elimination and eradication in Africa should consider a strategy that targets all Plasmodium species in parallel in asymptomatic and symptomatic infections based on accurate active case detection using highly sensitive molecular tools. ${ }^{16}$ It is essential for the scientific community to work closely with National Malaria Control Programs (NMCPs) to achieve this goal. By obtaining data regarding all Plasmodium species in Africa, the impetus for guidance regarding the elimination and eradication strategies will allow NMCPs to retool their resources appropriately.

There are geospatial limits to the transmission dynamics of Plasmodium species that can be missed with passive data 
collection, which is unable to detect submicroscopic and asymptomatic infections and how they contribute to transmission. ${ }^{17,18}$ The Botswana NMP is retooling to forge a collaboration to create elimination strategies and improve disease surveillance, monitoring, and evaluation. ${ }^{19}$ The collaboration will improve the human resources and technical capacity through joint activities with universities and research institutions. We report the data obtained using molecular tools through our close collaboration with the NMP of Botswana to detect active cases of the four Plasmodium species that are infectious to humans. We affirm the presence of $P$. vivax and nonfalciparum parasites in Botswana and the changed burden of $P$. falciparum in the country since the 2016 report.

\section{MATERIALS AND METHODS}

Study sites and population selection. The study sites were selected with the support of the NMP of the Ministry of Health and Wellness (MOHW) and Ministry of Education of Botswana, as reported previously. ${ }^{2}$ The sites were Okavango, Ngami, Chobe, Tutume, Francistown, Palapye, Serowe, Kweneng West, Kweneng East, and South East. We used a multistage sampling process to select the schools, clinics/ health posts, and participants. The Ministry of Basic Education primary school registers and MOHW clinic/health post registries within each district were used to select the schools and clinics involved in the study enrollment. ${ }^{2}$ Briefly, during the first stage, districts with known malaria transmission profiles based on the recommendations of the NMP were selected. During the second stage, towns within the district with variable malaria incidence rates were also selected to ensure heterogeneity within the districts. During the third stage, schools and clinics within each town/village were selected using a twostage clustering approach based on the population density and adequate cross-sectional representation of the communities to avoid any bias. During the final stage, participants were assigned numbers and enrolled after obtaining informed parental consent and consent from the heads of schools and clinics/health posts. The total number of samples derived for each district/town was in direct proportion to the estimated population density. We enrolled 1,645 children 2 to 13 years of age between December 2015 and February 2017.

Ethical statement. The study was approved by the MOHW Ethical Committee of Botswana and the Institutional Review Board of the University of Botswana (permit no. UBR/RES/ $\mathrm{IRB} / \mathrm{BIO} / 062)$. All parents/guardians provided informed consent on behalf of all participants. When necessary, assent was also obtained from the child before a sample was collected.

Sample size. The sample size was calculated as previously described. $^{2}$

Selection of subjects. Community mobilization was performed at Kgotla meetings (community meetings with elders of the community), clinics/health posts, and parent-teacher association meetings before enrollment. Children 5 to 9 years of age were enrolled from primary schools. Children 2 to 4 years of age and 10 to 13 years of age were enrolled from the child welfare clinics. Participants were enrolled only if they had no self-reported clinical symptoms.

Written informed consent for participation in the study was obtained in Setswana or English from parents or guardians for each prospective participant before enrollment. Emphasis was placed on the fact that the participants were free to participate or decline participation without any effect on their medical care.

Blood sample collection and baseline demographics. Venous blood samples (1.0-1.5 mL) were collected into EDTA tubes. Hemoglobin concentrations $(\mathrm{g} / \mathrm{dL})$ were checked immediately using a Hemocue 201 device (Angelholm, Sweden), and the results were provided to the participants' caregivers. Participants found to have moderate or severe anemia were referred for further investigation and treatment. Fever was defined as a tympanic temperature $\geq 37.8^{\circ} \mathrm{C}$ at the time of sample collection; asymptomatic participants were those without fever (tympanic temperature $<37.8^{\circ} \mathrm{C}$ ) and without a history of fever during the preceding 72 hours. Sample collections were timed to include the onset and peak of the malaria transmission season.

The samples were transported in cooler boxes within 3 hours of collection to the district hospital. Blood was separated by centrifugation at $5,000 \mathrm{rpm}$ for 5 minutes into plasma and cell pellets. These were stored at $-80^{\circ} \mathrm{C}$ until they were analyzed.

Demographic variables that were recorded at the time of blood sample collection were age, sex, ethnicity, height, weight, tympanic temperature, place of residence, travel history, and malaria diagnosis during the previous year. Participants who were underweight were referred for further management. Participants who were febrile were also referred to the outpatient department for further management.

Laboratory analysis. DNA extraction. Genomic DNA was extracted from the pelleted cells and purified using the Hamilton Star Microlab WorkStation (Hamilton Bonaduz AG, Bonaduz, Switzerland) robotic system. The instructions accompanying the Machery and Nagel (MN) Nucleospin 96 blood kit were followed to achieve the extraction of highquality DNA. This method uses silica membrane technology for binding DNA after lysis to facilitate purification. The starting blood sample was $200 \mu \mathrm{L}$ of the packed and thawed erythrocytes, and final DNA elution volume was $120 \mu \mathrm{L}$ sterile PCRgrade water. Then, $5 \mu \mathrm{L}$ of the extracted DNA was used for nested PCR and $2.5 \mu \mathrm{L}$ was used for quantitative PCR (qPCR).

Molecular detection of Plasmodium species All Plasmodium species were detected by modification of the doublenested PCR procedure targeting conserved species-specific regions of the18S small subunit of ribosomal RNA (18S ssRNA) as previously reported. ${ }^{2,20,21}$ For qPCR, the genusspecific primers and probes were those that have been previously described. ${ }^{21}$ The species-specific primers and probes for $P$. falciparum, $P$. vivax, $P$. ovale, and $P$. malariae were also published previously. ${ }^{22,23}$ Samples were screened during the qPCR for positivity and affirmed for species using nested PCR. All nested detection assays were single-plex and performed in a high-throughput 96-well plate (ABI GeneAmp 9700 PCR system; Applied BioSystems, Singapore), and the CFX96 Real-Time PCR detection system (Bio-Rad Laboratories, Pretoria, South Africa) was used for all qPCR assays. The kits used for nested PCR were the Qiagen PCR core kit (Qiagen, Valencia, CA); the KAPA PROBE FAST qPCR Master Mix (2X) (Sigma Inc., Cape Town, South Africa) or the abTES Malaria 5 qPCR II Kit (AITbiotech Pte Ltd, Singapore) were also used for PCR. Species-specific primers and probes were ordered from Eurogentec (Liege, Belgium). Standard controls of Plasmodium species 3D7 synchronized cultured parasites were obtained by cell sorting flow cytometry as described previously ${ }^{24}(10,000$ rings in $50 \mu \mathrm{L}$ of packed red blood cells). Details of the amplification reactions and 
cycling parameters for qPCR and nested PCR have been published previously. ${ }^{25}$

Statistical analysis. Data were entered in an Excel (Microsoft, Redmond, WA) data sheet. SPSS version 26 (release 2019; IBM Corp., Armonk, NY) was used for analysis. Descriptive statistics and appropriate measures of central tendency were provided for relevant demographic covariates. To describe differences between study sub-populations (e.g., different regions of residence with respect to the presence/ absence of Plasmodium infection, anemia, and/or age), continuous covariates grouped into categories were compared using binary and multinomial logistic regression. Odds ratios (ORs) were used for logistic regression analyses, and 95\% confidence intervals (Cls) were used for point estimates. Anemia was defined as hemoglobin $<11.0 \mathrm{~g} / \mathrm{dL}$. Statistical significance for all comparisons was set at $P<0.05$. Adjustment was not performed for multiple comparisons during this exploratory study.

\section{RESULTS}

Study population. A total of 1,645 children from 10 districts (Figure 1) were enrolled in the study. Data of 1,614 participants were available for analyses (Table 1). The median age of the participants was 5 years $\left(25^{\text {th }}\right.$ percentile, 3 years; $75^{\text {th }}$ percentile, 8 years). Participants from Tutume (median age, 3 years; $25^{\text {th }}$ percentile, 2 years; $75^{\text {th }}$ percentile, 5 years) were the youngest, followed by those from Kweneng East (median age, 5 years; $25^{\text {th }}$ percentile, 3 years; $75^{\text {th }}$ percentile, 8 years; $P=0.005$ ) and Serowe (median age, 4 years; $25^{\text {th }}$ percentile, 3 years; $75^{\text {th }}$ percentile, 7 years; $P=0.005$ ). Participants from Ngami (median age, 7 years; $25^{\text {th }}$ percentile, 5 years; $75^{\text {th }}$ percentile, 8 years) and Francistown (median age, 7 years; $25^{\text {th }}$ percentile, 4 years; $75^{\text {th }}$ percentile, 8 years) were older than the other participants $(P=0.004)$. The overall median hemoglobin concentration was $12.1 \mathrm{~g} / \mathrm{dL}$; however, anemia was present in the $<20^{\text {th }}$ percentile age group in all districts $(P<0.001)$ (Table 1). Of the patients 2 to 11 years of age (median age, 3.2 years; mean age, 3.95 years), 18.3\% had anemia. The risk of anemia decreased with age 5 years or older (OR, 0.26; $95 \% \mathrm{Cl}$, $0.197-0.34 ; P<0.001)$. Anemia was significantly related to the district of residence $(P<0.001)$ (Table 1$)$. Tutume $(4.5 \%)$ had the most cases of anemia $\left(10^{\text {th }}\right.$ percentile, $10.5 \mathrm{~g} / \mathrm{dL} ; 25^{\text {th }}$ percentile, $10.5 \mathrm{~g} / \mathrm{dL} ; P<0.001)$, followed by Okavango $(P=$ 0.09 ) and Francistown $(P=0.06)$ (Table 1).). The $10^{\text {th }}$ percentile and $25^{\text {th }}$ percentile hemoglobin concentrations $(\mathrm{g} / \mathrm{dL})$, respectively, were 10.2 and 10.7 for Francistown, 10.1 and 11.1 for Chobe, 10.6 and 11.3 for South East, 10.3 and 11.3 for Ngami, 10.5 and 11.4 for Kweneng East, and 10.8 and 11.3 for Serowe. Asymptomatic participants comprised $99.6 \%$ of the study population; $0.4 \%$ of the symptomatic participants had $P$. falciparum and were clustered mainly in Chobe. There was no difference in the sexes of participants who had Plasmodium infections and those who did not.

Plasmodium species. Data regarding Plasmodium parasites were available for 1,614 participants (Table 2). All four Plasmodium species infectious to humans were observed. The Plasmodium parasite infections with $P$. falciparum $(12.7 \%)$ and $P$. vivax (12.7\%) were similar, although the prevalence differed between districts (Table 2). The districts with the highest burden of Plasmodium infections were Kweneng East (11.96\%), South East (5.82\%), Ngami (2.6\%), Tutume (2.5\%), and Palapye (2.5\%); Kweneng West (0.80) and Okavango $(0.86 \%)$ had the least number of Plasmodium infections. All $P$. malariae and $P$. ovale (curtisi) infections were present as coinfections with $P$. falciparum or $P$. vivax and clustered in Kweneng East, South East, Tutume, and Palapye. $P$. vivax infections were strongly clustered in Kweneng East (5.6\%), South East (2.5\%), Ngami (1.3\%), and Tutume $(0.80 \%)$. According to a logistic regression analysis, the three districts with the highest risk of infection with $P$. vivax were Kweneng East (OR, 6.2; 95\% Cl, 2.94-13.1; $P<0.001)$, South East (OR, 5.6; 95\% Cl, 2.5-12.3; $P<0.001)$, and Ngami (OR, $5.1 ; 95 \% \mathrm{Cl}, 2.2-12.0 ; P<0.001)$. There was also a relatively small cluster at increased risk for infection in Kweneng West (OR, 2.7; 95\% Cl, 0.8-8.7) and Palapye (OR, 2.5; 95\% Cl, $0.9-6.8)$. The risk of infection with $P$. vivax decreased with age in Kweneng East (OR, 0.28; 95\% Cl, 0.15-0.52; $P<0.001)$, South East (OR, 0.31; 95\% Cl, 0.16-0.61; $P=0.001)$, and Ngami (OR, $0.35 ; 95 \% \mathrm{Cl}, 0.17-0.74 ; P=0.006)$. In contrast, all districts were significantly at risk for infection with $P$. falciparum $(P<0.001)$; however, participants 5 years or older were at decreased risk for infection in all regions except Chobe and Francistown. Interestingly, participants 5 years or older from Okavango were at increased risk for infection (OR, $2.5 ; 95 \% \mathrm{Cl},-0.94$ to $6.7 ; P=0.066)$.

\section{DISCUSSION}

The results of our survey demonstrate that the prevalence of asymptomatic $P$. falciparum and $P$. vivax increased significantly during 2016 to 2017, with $P$. vivax accounting for a significant proportion of the asymptomatic Plasmodium parasite population in Botswana. Interestingly, this observation

TABLE 1

Demographics of participants by district of residence

\begin{tabular}{|c|c|c|c|c|c|c|}
\hline \multirow[b]{2}{*}{ District (n)/Demographics } & \multirow[b]{2}{*}{$\begin{array}{c}\text { Age, median } \\
\left(25^{\text {th }}-75^{\text {th }} \text { percentile }\right)\end{array}$} & \multicolumn{2}{|c|}{$\operatorname{Sex}(\%)$} & \multirow[b]{2}{*}{$\begin{array}{l}\text { Hemoglobin, } \mathrm{g} / \mathrm{dL} \text {, median } \\
\left(25^{\mathrm{th}}-75^{\text {th }} \text { percentile }\right)\end{array}$} & \multirow[b]{2}{*}{$\begin{array}{l}\text { Total with } \\
\text { anemia, \% }\end{array}$} & \multirow[b]{2}{*}{$\begin{array}{l}\text { Total younger than } \\
5 \text { years, } \%\end{array}$} \\
\hline & & $\mathrm{F}$ & M & & & \\
\hline Okavango (194) & $6.0(4-8)$ & 11.9 & 12.2 & $12.3(11.5-13.1)$ & 1.4 & 3.8 \\
\hline Ngami (116) & $7.0(5-8)$ & 6.1 & 8.4 & $12.0(11.3-12.7)$ & 1.5 & 1.6 \\
\hline Chobe (162) & $4.5(4-8)$ & 10.2 & 9.9 & $12.0(11.1-12.7)$ & 2.0 & 5.0 \\
\hline Tutume (189) & $3.0(2-5)$ & 10.5 & 13.2 & $11.4(10.5-12.3)$ & 4.5 & 8.3 \\
\hline Francistown (102) & $7.0(4-8)$ & 6.3 & 6.3 & $11.9(10.7-12.8)$ & 1.7 & 1.6 \\
\hline Palapye (91) & $6.0(3-7)$ & 5.6 & 5.7 & $12.5(11.3-13.2)$ & 0.7 & 1.9 \\
\hline Serowe (79) & $4.0(4-8)$ & 5.2 & 4.6 & $12.3(11.7-13.6)$ & 0.2 & 2.5 \\
\hline Kweneng West (148) & $6.0(4-8)$ & 2.7 & 3.3 & $12.2(11.8-12.7)$ & 0.3 & 1.0 \\
\hline Kweneng East (427) & $5.0(3-8)$ & 28.8 & 24.1 & $12.2(11.4-12.9)$ & 3.9 & 13.1 \\
\hline South East (206) & $4.0(3-8)$ & 12.7 & 12.5 & $12.1(11.3-12.9)$ & 2.0 & 6.6 \\
\hline
\end{tabular}


TABLE 2

Prevalence of Plasmodium species detected per district

\begin{tabular}{|c|c|c|c|c|c|c|c|c|c|c|c|c|c|}
\hline \multirow[b]{2}{*}{ District } & \multirow{2}{*}{$\frac{\begin{array}{c}\text { Samples } \\
\text { tested }\end{array}}{\mathrm{N}}$} & \multicolumn{9}{|c|}{ Type and number of Plasmodium species detected } & \multirow{2}{*}{$\begin{array}{c}\begin{array}{c}\text { Total from } \\
\text { clinics }\end{array} \\
\mathrm{N}\end{array}$} & \multirow{2}{*}{$\begin{array}{c}\begin{array}{c}\text { Total from } \\
\text { schools }\end{array} \\
\mathrm{N}\end{array}$} & \multirow{2}{*}{$\begin{array}{c}\begin{array}{c}\text { Parasite prevalence/ } \\
\text { district }\end{array} \\
\%\end{array}$} \\
\hline & & Pf & Pv & $P m$ & Po & $P v+P f$ & $P f+P m$ & $P f+P o$ & $P o+P m$ & $P v+P m$ & & & \\
\hline Okavango & 194 & 6 & 8 & 0 & 0 & 0 & 0 & 0 & 0 & 0 & 56 & 138 & 0.86 \\
\hline Ngami & 116 & 17 & 21 & 0 & 0 & 4 & 0 & 0 & 0 & 0 & 26 & 90 & 2.6 \\
\hline Chobe & 162 & 17 & 10 & 0 & 0 & 3 & 0 & 0 & 0 & 0 & 89 & 73 & 1.86 \\
\hline Tutume & 189 & 14 & 13 & 3 & 2 & 1 & 3 & 2 & 2 & 0 & 150 & 39 & 2.5 \\
\hline Francistown & 102 & 11 & 5 & 0 & 0 & 2 & 0 & 0 & 0 & 0 & 27 & 75 & 1.1 \\
\hline Palapye & 91 & 15 & 9 & 3 & 3 & 1 & 3 & 3 & 3 & 0 & 31 & 60 & 2.5 \\
\hline Serowe & 79 & 13 & 4 & 0 & 0 & 0 & 0 & 0 & 0 & 0 & 43 & 36 & 1.0 \\
\hline Kweneng West & 48 & 8 & 5 & 0 & 0 & 0 & 0 & 0 & 0 & 0 & 17 & 31 & 0.80 \\
\hline Kweneng East & 427 & 71 & 90 & 3 & 2 & 21 & 2 & 1 & 2 & 1 & 237 & 190 & 11.96 \\
\hline South East & 206 & 33 & 40 & 3 & 3 & 6 & 3 & 3 & 3 & 0 & 117 & 89 & 5.82 \\
\hline Total & 1614 & 205 & 205 & 12 & 11 & 38 & 11 & 9 & 10 & 1 & 793 & 821 & \\
\hline$\%$ & 100 & 12.7 & 12.7 & 0.74 & 0.68 & 2.35 & 0.68 & 0.56 & 0.62 & 0.06 & 49.1 & 50.9 & \\
\hline
\end{tabular}

was affirmed by the clinical outbreak that led to the conclusion by the $\mathrm{WHO}$, during their assessment, that the malaria elimination agenda of Botswana was off course. ${ }^{6}$ Kweneng East and South East continue to be hotspots for asymptomatic $P$. vivax infections, ${ }^{2}$ which, unfortunately, appear to have spread together with $P$. falciparum into all districts. The presence of significant asymptomatic infections in the population has important implications for strategies and efforts to eliminate malaria. It has been established that $\geq 75 \%$ of Plasmodium infections are asymptomatic ${ }^{26}$; however, the strategic approach to interrupt Plasmodium transmission largely targets symptomatic infections because data from malaria control programs are accrued from passive rather than active case detection. ${ }^{18}$ Asymptomatic infections can be symptomatic during the short term or can remain for long periods $^{27,28}$ before becoming symptomatic. ${ }^{29}$ The caveat is that the majority of asymptomatic infections are also submicroscopic. $^{17,30,31}$ Therefore, only highly sensitive molecular tools can effectively assess their actual extent. ${ }^{32,33}$ It is necessary for malaria elimination programs to change their approach from passive to active case detection with molecular tools, ${ }^{34,35}$ which have the added benefit of being able to detect all Plasmodium parasites. ${ }^{36}$

An argument against the detection of asymptomatic infections in NMCPs is based on whether they contribute substantially to transmission. There are transmission hotspots for asymptomatic infections, ${ }^{37,38}$ as have been observed for $P$. vivax and $P$. falciparum, thereby affirming that they do contribute to transmission. It has also been observed that some submicroscopic infections may never progress to symptomatic infections and subtly contribute to transmission. ${ }^{18}$ There are variations in transmission patterns that are geospatially defined in a country, and these variations need to be understood. According to our report in $2016,{ }^{2}$ Kweneng East, South East, and Tutume were the major hotspots for $P$. vivax infection; however, a new locus of infection for $P$. vivax has emerged in Ngami in the north. Investigations of the Duffy antigen phenotype has been initiated to understand the observed trend.

It is evident that transmission in Okavango has decreased for children younger than 5 years and increased for children 5 years or older. However, it is not clear what the exact reasons are for this observed pattern of infection in this region. Perhaps the persistent efforts by the NMP to mitigate transmission in the district by indoor residual spraying may be a contributory factor. Loci of infections have clearly shifted to the south and southeastern districts, and new strategies are required. The spread of asymptomatic $P$. vivax in the country, including the north, is worrisome because it has significant implications for the elimination agenda because hypnozoites can sustain transmission by serving as infection reservoirs. ${ }^{37,39}$ This also applies to $P$. ovale. ${ }^{13}$ The data regarding Duffy negativity in the population are currently unavailable; however, there is the likelihood that some of the $P$. vivax infections will be Duffynegative, which will sustain the spread. Therefore, the presence of $P$. vivax and $P$. ovale makes it imperative to implement clear plans regarding the use of primaquine in Botswana. Primaquine has a spectrum of antimalarial activities that leads to the radical cure of hypnozoite stages and sterilization of gametocytes in P. falciparum. ${ }^{40}$ We previously reported that

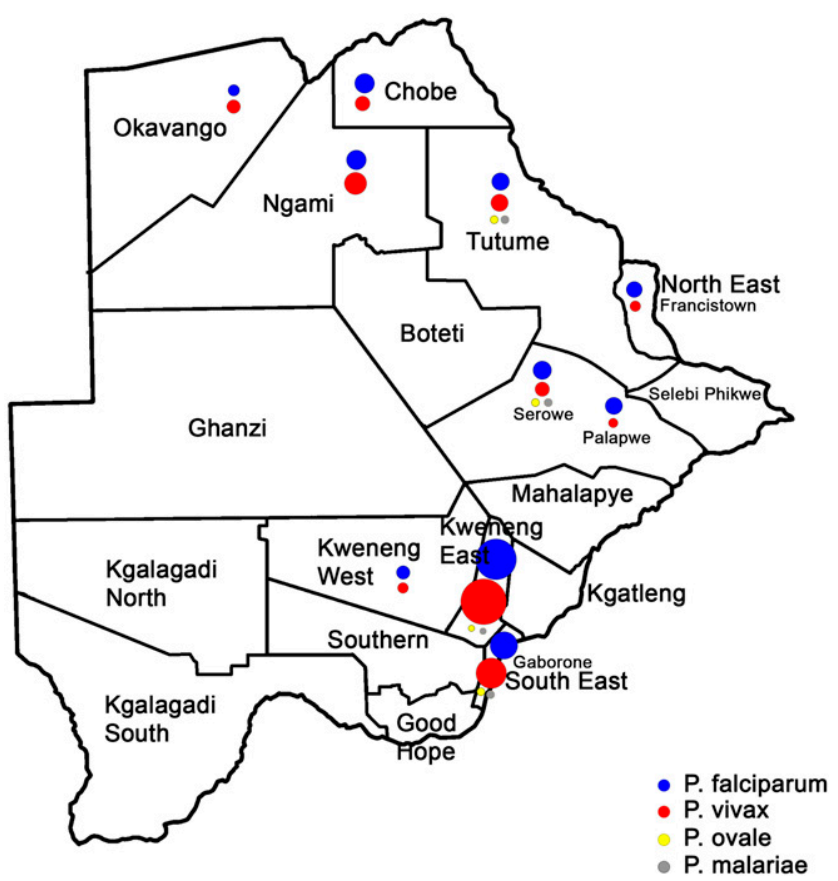

Figure 1. Prevalence of Plasmodium species by district. 
the G6PD deficiency in Botswana based on the hemizygous male frequency was $2.30 \%(95 \% \mathrm{Cl}, 1.77-2.83)$, and that the overall frequency of G6PD-deficient genotype A (hemizygote and homozygote genotypes only) was $1.26 \%(95 \% \mathrm{Cl}$, 0.86-1.66); these rates should be considered when deciding to implement the use of primaquine in the country. ${ }^{41} P$. vivax has been labeled as benign, although there is ample evidence to the contrary because of its association with severe malaria. ${ }^{42}$ Perhaps the lack of hyperparasitemia observed with $P$. vivax infections because of the preference for reticulocytes may be a contributing factor. ${ }^{43}$ Recent reports of the presence of $P$. vivax in the bone marrow present the hematopoietic system as a probable hiding place, in addition to the liver, thereby limiting vascular detection by methods other than PCR in nonendemic areas. ${ }^{43,44}$ What we have observed in Botswana can happen in other African countries that have reported the presence of $P$. vivax within the population; therefore, serious attention to this matter is required. An important observation is the level of anemia, which was severer for those younger than 5 years. This observation was related to the district and age, but not the Plasmodium infection status; therefore, these anemia cases are more likely to be related to the nutritional status. Nevertheless, we cannot exclude the contribution of existing coinfections. Because anemia can influence infections, and vice versa, particular attention by the NMP is required. ${ }^{45}$

In conclusion, we have shown that $P$. vivax is spreading along with $P$. falciparum in the asymptomatic population in Botswana. Local factors that affect transmission dynamics need to be carefully investigated in Botswana and other African countries. To achieve the elimination of malaria, the NMP in Botswana needs to integrate molecular tools in its strategic planning.

Received January 21, 2021. Accepted for publication March 9, 2021.

Published online May 3, 2021.

Acknowledgments: We acknowledge the staff of Botswana NMP who assisted with the field work and the assistance of WWARN in forging the collaboration with $A^{*}$ STAR, Singapore.

Financial support: This work was supported by the Global Health Institute, Merck KGaA, Darmstadt, Germany.

Authors' addresses: Thato Motshoge, University of Botswana, Gaborone, Botswana, E-mail: motshogethato@yahoo.com. Laurent Renia, A*STAR Infectious Disease Laboratory, A*STAR, Biopolis, Singapore, E-mail: renia_laurent@immunol.a-star.edu.sg. Daniel Hosea Haiyambo, University of Namibia, School of Medicine, Windhoek, Namibia, E-mail: dhaiyambo@unam.na. Ruth Ayanful-Torgby, Biomedical and Public Health Research Unit, Council for Scientific and Industrial Research-Water Research Institute, Council Close, Accra, Ghana, E-mail: ruthmine2@gmail.com. Larysa Aleksenko, University of Lund, Lund, Sweden, E-mail: larysa.aleksenko@med.lu.se. Davies Ntebela, National Malaria Program, Ministry of Health and Wellness, Gaborone, Botswana, E-mail: dntebela834@gmail.com. Benoit Malleret, Department of Microbiology and Immunology, Yong Loo Lin School of Medicine, National University of Singapore, National University Health System, Singapore, E-mail: benoit_malleret@ nus.edu.sg. Elias Peloewetse, University of Botswana, Gaborone, Botswana, E-mail: pelowee@mopipi.ub.bw. Giacomo Maria Paganotti, Botswana-University of Pennsylvania Partnership, Gaborone, Botswana, Division of Infectious Diseases, Perelman School of Medicine, University of Pennsylvania, Philadelphia, PA, and Department of Biomedical Sciences, Faculty of Medicine, University of Botswana, Gaborone, Botswana, E-mail: paganottig@bup.org.bw. Isaac K. Quaye, Regent University College of Science and Technology, Dansoman, Accra, Ghana, E-mail: isaac.quaye@regent.edu.gh.

This is an open-access article distributed under the terms of the Creative Commons Attribution (CC-BY) License, which permits unrestricted use, distribution, and reproduction in any medium, provided the original author and source are credited.

\section{REFERENCES}

1. Motlaleng $M$ et al., 2018. Driving towards malaria elimination in Botswana by 2018: progress on case-based surveillance, 2013-2014. Public Health Action 8: S24-S28.

2. Motshoge $T$ et al., 2016. Molecular evidence of high rates of asymptomatic $P$. vivax infection and very low $P$. falciparum malaria in Botswana. BMC Infect Dis 16: 520-016-1857-8.

3. Lindblade KA, Kachur SP, 2020. Opportunities for subnational malaria elimination in high-burden countries. Am J Trop Med Hyg 103: 2153-2154.

4. Lover AA, Baird JK, Gosling R, Price RN, 2018. Malaria elimination: time to target all species. Am J Trop Med Hyg 99: 17-23.

5. Hamre KES, Hodges JS, Ayodo G, John CC, 2020. Lack of consistent malaria incidence hotspots in a highland Kenyan area during a 10-year period of very low and unstable transmission. Am J Trop Med Hyg 103: 2198-2207.

6. WHO, 2019. The E-2020 Initiative of 21 Malaria-eliminating Countries: 2019 Progress Report. Available at: https://www.who. int/malaria/publications/atoz/e-2020-progress-report-2019/ en/.

7. Feachem RGA et al., 2019. Malaria eradication within a generation: ambitious, achievable, and necessary. Lancet 394: 1056-1112.

8. Ashley EA, Phyo AP, Carrara VI, Tun KM, Nosten F, Smithuis F, White NJ, 2019. Plasmodium vivax relapse rates following Plasmodium falciparum malaria reflect previous transmission intensity. J Infect Dis 220: 100-104.

9. Yman V et al., 2019. Persistent transmission of Plasmodium malariae and Plasmodium ovale species in an area of declining Plasmodium falciparum transmission in eastern Tanzania. PLoS Negl Trop Dis 13: e0007414.

10. Zimmerman PA, 2017. Plasmodium vivax infection in Duffynegative people in Africa. Am J Trop Med Hyg 97: 636-638.

11. Snounou G, Pinheiro L, Goncalves A, Fonseca L, Dias F, Brown KN, do Rosario VE, 1993. The importance of sensitive detection of malaria parasites in the human and insect hosts in epidemiological studies, as shown by the analysis of field samples from Guinea Bissau. Trans R Soc Trop Med Hyg 87: 649-653.

12. Grande R, Antinori S, Meroni L, Menegon M, Severini C, 2019. A case of Plasmodium malariae recurrence: recrudescence or reinfection? Malar J 18: 169-019-2806-y.

13. Sutherland CJ, 2016. Persistent parasitism: the adaptive biology of malariae and ovale malaria. Trends Parasitol 32: 808-819.

14. Zhou Zet al., 2016. Assessment of submicroscopic infections and gametocyte carriage of Plasmodium falciparum during peak malaria transmission season in a community-based crosssectional survey in western Kenya, 2012. Malar J 15: 421-0161482-4.

15. Tadesse FG et al., 2018. The relative contribution of symptomatic and asymptomatic Plasmodium vivax and Plasmodium falciparum infections to the infectious reservoir in a low-endemic setting in Ethiopia. Clin Infect Dis 66: 1883-1891.

16. Sturrock HJ, Hsiang MS, Cohen JM, Smith DL, Greenhouse B, Bousema T, Gosling RD, 2013. Targeting asymptomatic malaria infections: active surveillance in control and elimination. PLoS Med 10: e1001467.

17. Tadesse FG, Pett H, Baidjoe A, Lanke K, Grignard L, Sutherland C, Hall T, Drakeley C, Bousema T, Mamo H, 2015. Submicroscopic carriage of Plasmodium falciparum and Plasmodium vivax in a low endemic area in Ethiopia where no parasitaemia was detected by microscopy or rapid diagnostic test. Malar J 14: 303-015-0821-1.

18. Bousema T, Okell L, Felger I, Drakeley C, 2014. Asymptomatic malaria infections: detectability, transmissibility and public health relevance. Nat Rev Microbiol 12: 833-840.

19. Kgoroebutswe TK et al., 2020. Vector control for malaria elimination in Botswana: progress, gaps and opportunities. Malar $J$ 19: 301-020-03375-6.

20. Snounou G, Viriyakosol S, Zhu XP, Jarra W, Pinheiro L, do Rosario VE, Thaithong S, Brown KN, 1993. High sensitivity of detection 
of human malaria parasites by the use of nested polymerase chain reaction. Mol Biochem Parasitol 61: 315-320.

21. Haiyambo DH et al., 2019. Glucose-6-phosphate dehydrogenase deficiency genotypes and allele frequencies in the Kavango and Zambezi regions of northern Namibia. Trans $R$ Soc Trop Med Hyg 113: 483-488.

22. Perandin F et al., 2004. Development of a real-time PCR assay for detection of Plasmodium falciparum, Plasmodium vivax, and Plasmodium ovale for routine clinical diagnosis. J Clin Microbiol 42: 1214-1219.

23. Rougemont M, Van Saanen M, Sahli R, Hinrikson HP, Bille J, Jaton $\mathrm{K}, 2004$. Detection of four Plasmodium species in blood from humans by $18 \mathrm{~S}$ rRNA gene subunit-based and speciesspecific real-time PCR assays. J Clin Microbiol 42: 5636-5643.

24. Malleret B, Claser C, Ong AS, Suwanarusk R, Sriprawat K, Howland SW, Russell B, Nosten F, Renia L, 2011. A rapid and robust tri-color flow cytometry assay for monitoring malaria parasite development. Sci Rep 1: 118

25. Haiyambo DH, Uusiku P, Mumbengegwi D, Pernica JM, Bock R, Malleret B, Renia L, Greco B, Quaye IK, 2019. Molecular detection of $P$. vivax and $P$. ovale foci of infection in asymptomatic and symptomatic children in northern Namibia. PLoS Negl Trop Dis 13: e0007290.

26. Greenwood BM, 1987. Asymptomatic malaria infections-do they matter? Parasitol Today 3: 206-214.

27. Njama-Meya D, Kamya MR, Dorsey G, 2004. Asymptomatic parasitaemia as a risk factor for symptomatic malaria in a cohort of Ugandan children. Trop Med Int Health 9: 862-868.

28. Tran TM, Aghili A, Li S, Ongoiba A, Kayentao K, Doumbo S, Traore B, Crompton PD, 2014. A nested real-time PCR assay for the quantification of Plasmodium falciparum DNA extracted from dried blood spots. Malar J 13: 393-2875-13-393.

29. Douglas AD, Andrews L, Draper SJ, Bojang K, Milligan P, Gilbert SC, Imoukhuede EB, Hill AV, 2011. Substantially reduced prepatent parasite multiplication rates are associated with naturally acquired immunity to Plasmodium falciparum. J Infect Dis 203: 1337-1340.

30. Belizario VY, Saul A, Bustos MD, Lansang MA, Pasay CJ, Gatton M, Salazar NP, 1997. Field epidemiological studies on malaria in a low endemic area in the Philippines. Acta Trop 63: 241-256.

31. Okell LC, Bousema T, Griffin JT, Ouedraogo AL, Ghani AC, Drakeley CJ, 2012. Factors determining the occurrence of submicroscopic malaria infections and their relevance for control. Nat Commun 3: 1237

32. Bejon P, Andrews L, Hunt-Cooke A, Sanderson F, Gilbert SC, Hill AV, 2006. Thick blood film examination for Plasmodium falciparum malaria has reduced sensitivity and underestimates parasite density. Malar J 5: 104-2875-5-104.

33. Mosha JF et al., 2013. Epidemiology of subpatent Plasmodium falciparum infection: implications for detection of hotspots with imperfect diagnostics. Malar J 12: 221-2875-12-221.

34. Cotter C, Sturrock HJ, Hsiang MS, Liu J, Phillips AA, Hwang J, Gueye CS, Fullman N, Gosling RD, Feachem RG, 2013. The changing epidemiology of malaria elimination: new strategies for new challenges. Lancet 382: 900-911.

35. Cotter C, Sudathip P, Herdiana H, Cao Y, Liu Y, Luo A, Ranasinghe N, Bennett A, Cao J, Gosling RD, 2017. Piloting a programme tool to evaluate malaria case investigation and reactive case detection activities: results from 3 settings in the Asia Pacific. Malar J 16: 347-017-1991-9.

36. Snounou G, Viriyakosol S, Jarra W, Thaithong S, Brown KN, 1993. Identification of the four human malaria parasite species in field samples by the polymerase chain reaction and detection of a high prevalence of mixed infections. Mol Biochem Parasitol 58: 283-292.

37. Alves FP, Durlacher RR, Menezes MJ, Krieger H, Silva LH, Camargo EP, 2002. High prevalence of asymptomatic Plasmodium vivax and Plasmodium falciparum infections in native Amazonian populations. Am J Trop Med Hyg 66: 641-648.

38. Bousema $T$ et al., 2010. Identification of hot spots of malaria transmission for targeted malaria control. $J$ Infect Dis 201: 1764-1774.

39. Howes RE et al., 2015. Plasmodium vivax transmission in Africa. PLoS Negl Trop Dis 9: e0004222.

40. Recht J, Ashley EA, White NJ, 2018. Use of primaquine and glucose-6-phosphate dehydrogenase deficiency testing: divergent policies and practices in malaria endemic countries. PLoS Negl Trop Dis 12: e0006230.

41. Motshoge T et al., 2018. Prevalence of G6PD deficiency and associated haematological parameters in children from Botswana. Infect Genet Evol 63: 73-78.

42. Naing C, Whittaker MA, Nyunt Wai V, Mak JW, 2014. Is Plasmodium vivax malaria a severe malaria? A systematic review and meta-analysis. PLoS Negl Trop Dis 8: e3071.

43. Silva-Filho JL, Lacerda MVG, Recker M, Wassmer SC, Marti M, Costa FTM, 2020. Plasmodium vivax in hematopoietic niches: hidden and dangerous. Trends Parasitol 36: 447-458.

44. Obaldia N, 3rd et al., 2018. Bone marrow is a major parasite reservoir in Plasmodium vivax infection. MBio 9. doi: 10.1128/ mBio.00625-18.

45. White NJ, 2018. Anaemia and malaria. Malar J 17: 371-0182509-9. 